\title{
On the shoulders of data
}

\author{
Over the past decades, the availability of new methods and digitization has dramatically changed how scientific \\ data are recorded, stored and analysed. This has enabled researchers to pull together the data underlying single \\ research efforts into larger standardized datasets for reuse. The publication of these datasets - in the Resource \\ format in our pages - represents a contribution of exceptional value to the scientific community.
}

$\mathrm{T}$ he advent of online publication of research articles has long given the community the ability to directly link the description and interpretation of their research in the article to the underlying data. Sharing data enables reuse in novel research and thus not only enables reproducibility, but also accelerates scientific progress whilst reducing its cost. But modern forms of publishing offer more than just making the data that underpin the reported results available. Untethered from single projects, large collections of data can constitute a resource worth publishing in their own right.

Undoubtedly, there is significant value in data, particularly when the data are large, costly or difficult to collect. Consequently, publishing these data is a significant community service. At Nature Human Behaviour, we welcome such contributions in the form of Resources, that is, large datasets or tools of broad utility, interest and significance to the community. Two recent examples of Resources published in our pages exemplify how collections of data constitute valuable assets to future research.

Kai Miller published a Resource consisting of human electrocorticographic data and example analyses (https:// doi.org/10.1038/s41562-019-0678-3). Electrocorticographic data is recorded by implanting electrodes onto the human brain. This procedure delivers data of high temporal and high spatial resolutions, a combination that many other approaches to neuroimaging lack. However, as an invasive measure, it is only undertaken when clinically necessary, for example when preparing patients with epilepsy for surgery; this has historically limited the availability of these valuable data. Miller's publication consists of a sizeable collection of 204 datasets from 16 different experiments, outstripping the size of most prior studies. Publishing data and analysis scripts prepared in an accessible and standardized format allows other scientists, including those without access to a clinic, to test their own research questions and thus amplifies the benefits reaped from the time and data volunteered by the patients.

Another Resource recently published in our pages is the result of a large, ongoing collaboration project, led by Dobromir Rahnev (https://doi.org/10.1038/s41562019-0813-1). The Confidence Database contains an array of studies that explore how humans form meta-cognitive judgments about their performance. At the time of publication, the growing database contained data from more than 8,700 participants, including more than 4 million individual responses. Such a wealth of data significantly increases the robustness and generalizability of analyses. By providing an unprecedented wealth of data on human confidence judgments, the Resource offers a substantive increase in confidence in the reliability of the results. Moreover, the multitude of paradigms and experimental conditions allows tests for generalization of principles that remain unfeasible on the back of single studies.

These and all other Resource articles in Nature Human Behaviour are formally structured like research articles, linking to the deposited data and/or code. The manuscripts explain the purpose and utility of the resource and contain detailed Methods. Data must be prepared to be accessible and clearly structured. Comprehensive, transparent documentation of data and code are required, and care must be taken to protect the rights of any participants who volunteered their data. Resources are peer-reviewed.

Research is a collective, cumulative endeavour in which individuals' ideas and findings inspire others to follow in their footsteps and build on their achievements. Progress in science thrives not only on reading about the ideas and results of others. Standardized pooled datasets, databases, and tools can be of exceptional value and merit publication in their own right. Projects that lead to these precious contributions are laudable efforts and we welcome submission of Resources that will advance research in our broad community of human behaviour researchers.

Published online: 17 March 2020 https://doi.org/10.1038/s41562-020-0847-4 